\title{
Self-trapped states in proteins?
}

\author{
Robert H Austin ${ }^{1}$, Aihua $\mathrm{Xie}^{2}$, Lex van der Meer ${ }^{3}$, Michelle Shinn ${ }^{4}$ and \\ George Neil ${ }^{4}$ \\ ${ }^{1}$ Department of Physics, Princeton University, Princeton, NJ 08544, USA \\ 2 Department of Physics, Oklahoma State University, Stillwater, OK 74078, USA \\ ${ }^{3}$ FOM Institute for Plasma Physics, Edisonbaan 14, Nieuwegein, The Netherlands \\ ${ }^{4}$ Jefferson Laboratory, Newport News, VA 23606, USA
}

Received 8 November 2002

Published 28 April 2003

Online at stacks.iop.org/JPhysCM/15/S1693

\begin{abstract}
We show here that the temperature dependence of the amide Iband of myoglobin shows evidence for a low-lying self-trapped state at $6.15 \mu \mathrm{m}$. We have conducted a careful set of picosecond pump-probe experiments providing results as a function of temperature and wavelength and show that this lowlying state has a $30 \mathrm{ps}$ lifetime at $50 \mathrm{~K}$, much longer than the relaxation time of the main amide I band at $50 \mathrm{~K}$. Fits of the temperature dependence of thermal occupation of this state yield the result that it lies $280 \mathrm{~K}$ below the main amide I band. Since the gap energy of this state is approximately equal to room temperature, this self-trapped state can act as a transient store of vibrational energy at physiological temperatures in biomolecules and can help to direct the path of energy flow in a biomolecule under biological conditions.

(Some figures in this article are in colour only in the electronic version)
\end{abstract}

Although it is no longer fashionable to speak of proteins as systems resembling condensed matter ensembles of collectively interacting modes, there still remain basic questions of protein dynamics which are probably not easily addressed by either all-atom computer simulations or $\mathrm{x}$-ray crystallography. For example, many proteins undergo major conformational transitions when they bind small molecules, or when a molecular group within the protein undergoes a (for example) light-driven isomeric change. This collective response to a relatively small perturbation is inherently anharmonic and diagnostic of the rough energy landscape that seems to lie at the heart of our understanding of protein action. Exploration of this rough energy landscape requires not only studies of perturbations around the equilibrium set of conformational states of the protein, which only probe small deviations from the thermal mean, but also non-perturbative excitations far from the thermal mean. Such non-perturbative studies probe the biologically important anharmonic transitions between collective modes.

Given then that the collective dynamics far from thermal equilibrium is important in understanding protein action in a biological context, we can continue to be 'fashion victims' 


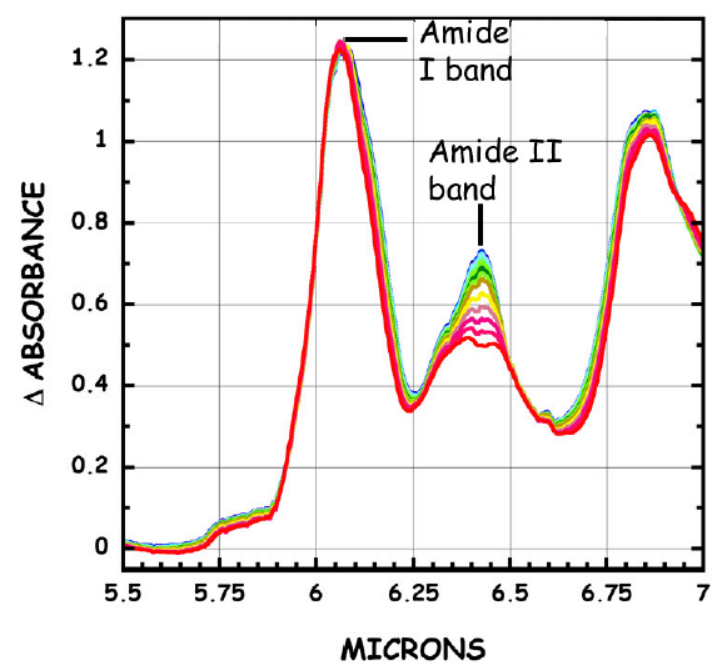

Figure 1. The temperature dependence of sperm whale MB (swMB) in the region $1800-1400 \mathrm{~cm}^{-1}$. The swMB was obtained from Sigma Chemicals (St Louis, MO) in lyophilized form. The powder was dissolved in $25 \mu \mathrm{l}$ of deuterium oxide at $10 \mathrm{mg} \mathrm{ml}^{-1}$ and then $75 \mu \mathrm{l}$ of deuterated glycerol was added to make a $75 \% \mathrm{~V} / \mathrm{V}$ solution. The swMB was put between a $\mathrm{CaF}_{2}$ pair of windows using a $12 \mu \mathrm{m}$ Mylar spacer and cooled in an Oxford Optistat helium cryostat. The sample remained at atmospheric pressure during the run. Infrared spectra were taken on a Perkin-Elmer FTIR spectrometer. In grey scale, the sense of the temperatures is from $5 \mathrm{~K}$ at the maximum absorbance to $300 \mathrm{~K}$ at the lowest absorbance. The colours of the curves are spectrally coded to correspond to temperature, with red $=300 \mathrm{~K}$, yellow $=180 \mathrm{~K}$ and blue $=5 \mathrm{~K}$.

and ask what major concept from condensed matter physics can help in understanding the dynamics in a complex system. We naturally turn to the concept of self-trapping, where a many-body system which has strong interactions with its internal degrees of freedom forms a new lower-lying state which is separated by a gap of energy $E$ from the set of degenerate energy levels for the degrees of freedom of the system if coupling interactions are turned off. Self-trapping results in the creation of a new state of the system which lies lower by $E$ than the non-interacting levels, where $E$ is roughly equal to the coupling strength $U_{i j}$ of the internal degrees of freedom.

It has long been proposed that certain secondary structures in proteins are candidates for exhibiting the formation of self-trapped states, such as the amide I band in $\alpha$-helix-containing proteins [1]. It has been proposed from studies of model hydrogen-bonding organic crystals such as acetanalide that the signature of such a self-trapped state is the appearance at low temperatures of a red-shifted band arising from the main set of states [2], and a recent paper suggests that self-trapping can occur in the extremely anharmonic $\mathrm{N}-\mathrm{H}$ modes of acetanalide [3]. However, the real question is whether such anharmonic effects exist in proteins.

The answer, surprisingly, seems to be yes. First, we discuss the static IR spectroscopy of a protein and show that there exist low-lying collective states. Figure 1 shows the IR absorbance spectrum of the predominantly $\alpha$-helix protein myoglobin as a function of temperature in the amide I and amide II regions of the infrared spectrum. There is a clear and strong temperature dependence of both the amide I and the amide II bands as a function of temperature. While the amide II band shows a increase of the band oscillator strength with decreasing temperature, the amide I band shows evidence for the formation at low temperatures of a band to the longwavelength side of the main amide I transition; the new state has an excitation energy of 


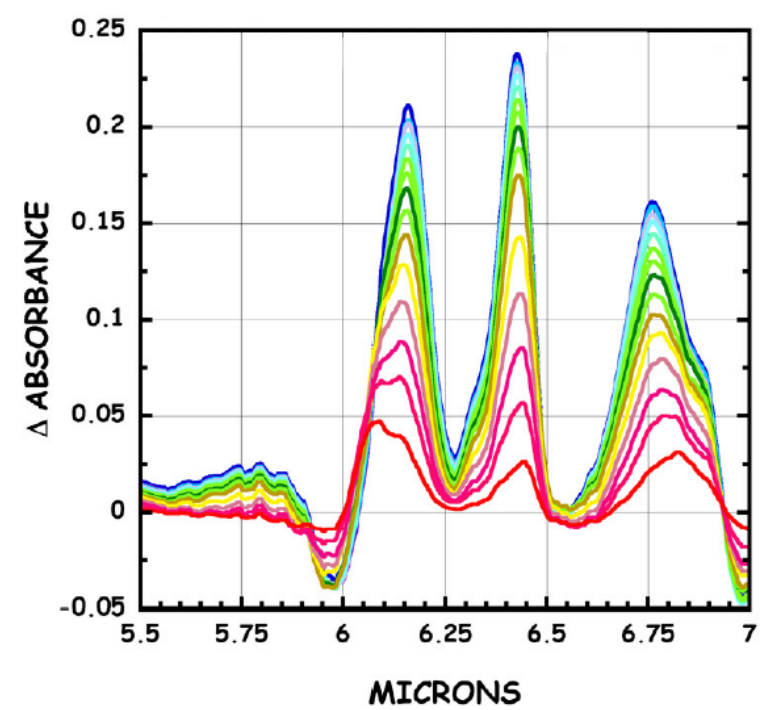

Figure 2. Difference spectra taken from figure 2. The $300 \mathrm{~K}$ spectrum of swMB was used as the base spectrum; we use the same colour scheme as in figure 2 to plot $\Delta$ absorbance $=$ (absorbance at temperature $T$ ) - (absorbance at $T=300 \mathrm{~K}$ ). In grey scale, the sense of the changes are from $5 \mathrm{~K}$ at maximum absorbance to $300 \mathrm{~K}$ at minimum absorbance for all peaks except that at 5.75 microns.

$6.15 \mu \mathrm{m}$ (or $1626 \mathrm{~cm}^{-1}$ ), as shown in figure 2. A similar temperature-dependent band near room temperature is also observed for model $\alpha$-helix peptides as discussed by the paper of Williams et al [4], which is consistent with our description of this band as due to a highly ordered $\alpha$-helix configuration of a polypeptide chain. In the case of the model polypeptide studied by Williams et al, the temperature dependence of the $6.15 \mu \mathrm{m}$ band is shifted to higher temperatures. We do not know the origin of this increase in the transition temperature and the increased cooperativity of the transition compared to that of myoglobin. It is interesting to note that the polypeptide studied by Williams et al was basically a small homopeptide (21 residues), consisting of almost exclusively alanine residues. Such a homogeneous polypeptide might actually be viewed as a peptide chain with fewer defects and meriting studies similar to those carried out here.

We fit the temperature dependence of the amide I two-state system to the $\operatorname{simple} \tanh (E / T)$ function expected for two states separated by a gap of energy $E$. Figure 1(B) shows the results of fitting the absorbance at $6.15 \mu \mathrm{cm}$ to a tanh function. The curve fitting shown in figure 3 yields that the state lies lower than the main amide I band by $E=280 \mathrm{~K}$, and thus at room temperature the transition is approximately its mid-point.

Next, we come to the critical question of dynamics. While the temperature-dependent static IR spectra are indicative of anharmonicity and the existence of thermally accessible lowlying states, only dynamic information can tell us whether these low-lying states do in fact have the anomalously long lifetime expected for a self-trapped state. Accordingly, we have carried out a series of picosecond-time-resolved pump-probe experiments using the free electron laser FELIX to determine the temperature dependence of the lifetime of this red-shifted state. In any pump-probe experiment in the infrared, and particularly if the band studied is a temperaturesensitive one, it is very important to closely control the heating of the sample to ensure that one is studying the dynamics of a cold molecule and not one heated during the measurement 


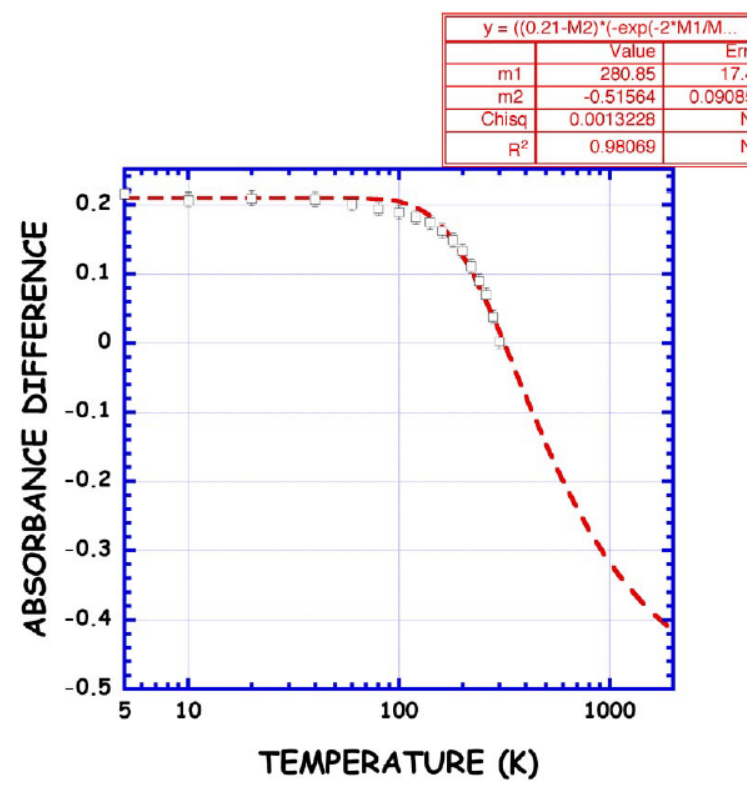

Figure 3. The curve fit using $\tanh (E / T)$ of the difference absorbance at $6.15 \mu \mathrm{m}$ versus $T$. The curve fitting program Kaleidagraph was used. The curve fit shown yields $E=280 \mathrm{~K}$.

process. This is extremely difficult to do because

(1) proteins are relatively harmonic systems and need high energies to show even small saturation signals in pump-probe experiments;

(2) the IR radiation at FELIX and at the Jefferson Laboratory comes in macropulses consisting of megahertz repetition rate micropulses of energy 1-10 $\mu \mathrm{J}$ each.

This means that the sample heats with a temperature increase of up to $100{ }^{\circ} \mathrm{C}$ during a macropulse and great care must be taken to ensure that data are taken not from the rear of a macropulse but rather from the front of the macropulse. We have devised two-box-car signal acquisition techniques which allow us to monitor the pump-probe signals both at the front and the rear of the macropulse so that this important aspect can be maintained.

This preliminary publication is based upon two separate experiments: initial temperaturedependent experiments were carried out at the Jefferson Laboratory free electron laser to determine the optimal configuration and test the stability of the mounting assembly for the Oxford flow cryostat; then the final runs were done at FELIX - where we took advantage of the tunability of the variable gap wrigglers. Pump-probe measurements were done using the same delay line compensation technique as used in our earlier work [5,6]. In this experiment we were careful to operate the Stanford boxcar amplifiers in the 'toggle' mode, which means that a synchronized set of shutters blocked the FEL pump beam on alternate macropulses and the boxcar amplifier subtracted the baseline from the running sum. Although we only use 50\% of the FEL pulses in this mode, there a very important advantage here in that slow DC drifts of the electronics and optics are nulled out, with the result that long relaxation times (tens of picoseconds) which require large movements of the delay line give stable baseline signals.

We carried out experiments at FELIX at two wavelengths: $6.01 \mu \mathrm{m}$ (the blue side of the amide I band where there is little temperature-dependent absorption) and at $6.15 \mu \mathrm{m}$ (the red side of the amide I band where the low-temperature band has a peak absorbance). Figure 4 shows the pump-probe result for $\mathrm{Mb}$ at $280 \mathrm{~K}$. There are four traces: front and rear, 6.02 and 
(A)

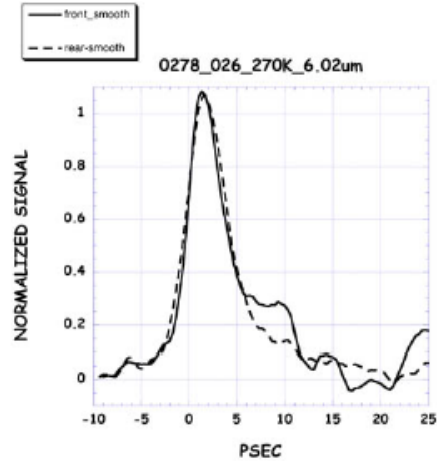

(B)

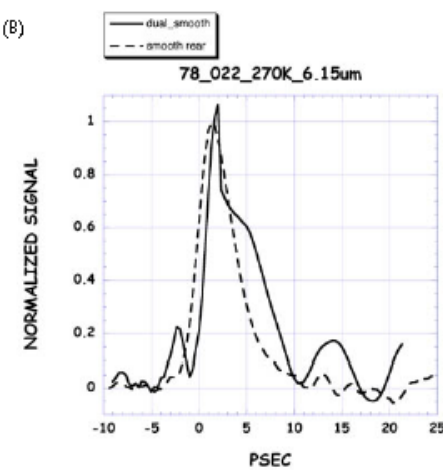

Figure 4. The $280 \mathrm{~K}$ pump-probe relaxation of $\mathrm{Mb}$ in the region of the amide I band at (A) $6.02 \mu \mathrm{m}$ and (B) $6.15 \mu \mathrm{m}$. 'front' refers to the first $0.5 \mu \mathrm{s}$ of the macropulse and 'rear' refers to the region from 1.5 to $2.0 \mu$ s of the macropulse.

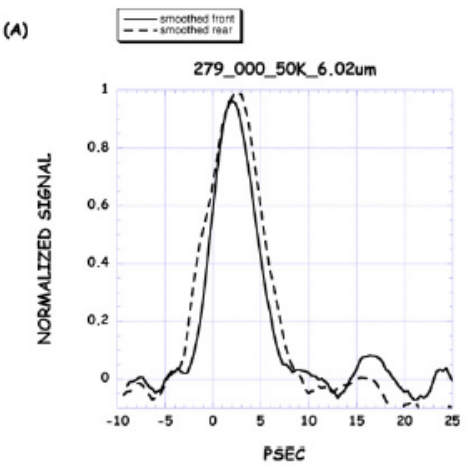

(B)

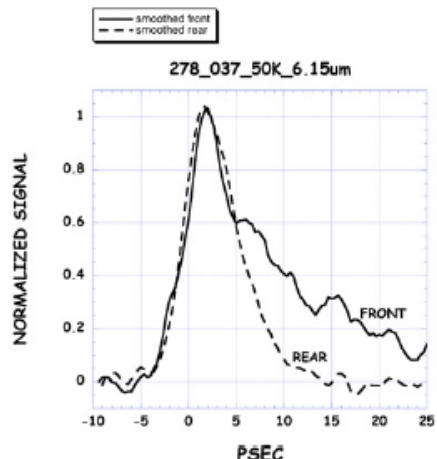

Figure 5. The $50 \mathrm{~K}$ pump-probe relaxation of $\mathrm{Mb}$ in the region of the amide I band at (A) $6.02 \mu \mathrm{m}$ and (B) $6.15 \mu \mathrm{m}$. 'front' refers to the first $0.5 \mu \mathrm{s}$ of the macropulse and 'rear' refers to the region from 1.5 to $2.0 \mu$ s of the macropulse.

$6.15 \mu \mathrm{m}$. Although there are some differences between the 6.02 and $6.15 \mu \mathrm{m}$ data, basically the four sets of data are the same at $280 \mathrm{~K}$.

However, at $50 \mathrm{~K}$ the results are quite different. Note that we did not attempt to go below $50 \mathrm{~K}$ in the pump-probe data because the rapidly decreasing specific heat of materials below $50 \mathrm{~K}$ make it problematic to determine the actual sample temperature even at the front of the macropulse given our $0.5 \mu$ s wide window. Figure 5 shows that at $50 \mathrm{~K}$ there are strong differences between the relaxation times at 6.02 and $6.15 \mu \mathrm{m}$. The $6.02 \mu \mathrm{m}$ relaxation rate is effectively the same at the front and the rear of the macropulse at $50 \mathrm{~K}$, while at $6.15 \mu \mathrm{m}$ the relaxation process is substantially slower at the front of the macropulse, where the sample is cold, compared to the rear of the macropulse, where the sample has heated by an estimated 50-100 K during the macropulse. Curve fitting of the relaxation rates yields that the lifetime of the $6.15 \mu \mathrm{m}$ band is approximately $30 \mathrm{ps}$, as opposed to the $5 \mathrm{ps}$ lifetime of the $6.02 \mu \mathrm{m}$ band.

We believe that the slowly relaxing signal corresponds to the intrinsic relaxation rate of the low-temperature band observed in static IR spectroscopy. If the low-temperature band does indeed correspond to a self-trapped state, then we would also expect the relaxation time of this self-trapped state to be long, as is observed. The implications of this self-trapping and 
energy storage in the delocalized amide I band of myoglobin are important: this opens up a new way to view the dynamics of coupled anharmonic systems in an intuitive way. Clearly a great number of other experiments need to be done before the full generality of this result can be understood.

\section{Acknowledgments}

We thank R van Buuren, P F M Delmee, W J Mastop and Th Ram for operation of FELIX, P F M Delmee for assistance in construction of the experiment, and W D Hoff for stimulating discussions. We especially thank Ian Bradley for developing much of the LabView code used in these experiments and for transporting a flow cryostat to the Jefferson Laboratory for initial runs there. This work was supported by the Office of Naval Research, and the Stichting voor Fundamenteel Onderzoek der Materie (FOM) provided the required beam time on FELIX.

\section{References}

[1] Davidov A S 1977 J. Theor. Biol. 66379

[2] Careri G, Buontempo U, Carta F, Gratton E and Scott A C 1983 Phys. Rev. Lett. 83304

[3] Edler J, Hamm P and Scott A C 2002 Phys. Rev. Lett. 88 067403(4)

[4] Williams S, Causgrove T P, Gilmanshin R, Fang K, Callender R H, Woodruff W H and Dyer R D 1996 Biochemistry 35 691-7

[5] Xie A, van der Meer A, Hoff W and Austin R H 2000 Phys. Rev. Lett. 84 5435-8

[6] Xie A, van der Meer A and Austin R H 2002 Phys. Rev. Lett. 88 018102(4) 